\title{
A Petrov-Galerkin method for integro-differential equations with a memory term
}

\author{
K. Mustapha ${ }^{1}$
}

(Received 29 July 2008; revised 16 December 2008)

\begin{abstract}
We investigate the numerical solution of an integro-differential equation with a memory term. For the time discretization we apply the continuous Petrov-Galerkin method considered by Lin et al. [SIAM J. Numer. Anal., 38, 2000]. We combined the Petrov-Galerkin scheme with respect to time with continuous finite elements for the space discretization and obtained a fully discrete scheme. We show optimal error bounds of the numerical solutions for both schemes, and compare our theoretical error bounds with the results of numerical computations.
\end{abstract}

http://anziamj.austms.org.au/ojs/index.php/ANZIAMJ/article/view/1382 gives this article, (c) Austral. Mathematical Soc. 2008. Published December 22, 2008. ISSN 1446-8735. (Print two pages per sheet of paper.) 


\section{Contents}

1 Introduction

C611

2 Stability results

C615

3 Error estimates

C618

4 Numerical experiments

C622

References

C624

\section{Introduction}

We study a class of numerical solutions for a linear integro-differential equation of the form

$$
u^{\prime}(t)+A u(t)+\int_{0}^{t} b(t, s) A u(s) d s=f(t), \quad t \in(0, T] \quad \text { with } u(0)=u_{0}
$$

where $\mathfrak{u}^{\prime}(t):=\frac{\partial u}{\partial t}(t), \mathfrak{u}(t)=\mathfrak{u}(x, t)$ and $f(t)=f(x, t)$ with $x \in \Omega(\Omega$ is a bounded domain subset of $\left.\mathbb{R}^{m}, m \geq 1\right)$. Here $A$ is a linear, positive definite, self-adjoint operator with domain $D(A)$ in a real Hilbert space $\mathbb{H}$. The solution $u$ and the source term $f$ take values in $\mathbb{H}$, and the initial data $u_{0}$ is an element of $\mathbb{H}$. We set $\|v\|_{p}=\left\|A^{p / 2} \mathcal{v}\right\|=\left\langle A^{p} \mathcal{v}, v\right\rangle^{1 / 2}$, where $\|\cdot\|$ is the norm and $\langle\cdot, \cdot\rangle$ is the inner product in $\mathbb{H}$.

For $0 \leq i+j \leq 1$ and for some $0<\alpha<1$, we assume that

$$
\left|\frac{\partial^{i+j}}{\partial t^{i} \partial s^{j}} b(t, s)\right| \leq C(t-s)^{\alpha-1} .
$$


Many have considered numerical methods of the problem (1). Typically, time discretization is performed by either using finite difference or continuous and discontinuous finite elements with a quadrature. Finite difference and discontinuous Galerkin in time, and finite elements in space have been discussed in the case of a smooth and non-smooth memory term (Larsson et al. [1] and Zhang [4]). Another approach using Laplace transform has been studied, for example, by McLean et al. [3].

In this article we solve the problem (1) using the Petrov-Galerkin method with respect to time (PGMT). Then we combine the PGMT with the finite elements discretization in space, which defines a fully discrete Petrov-Galerkin $(\mathrm{PG})$ finite element method (PGFEM) for (1). We derive the error estimates from both schemes. In addition, we present numerical evidence that our error bounds are optimal.

Unlike the standard Galerkin method, which is a special case of the PG method, the PG method allows the trial and test spaces to be different. The considered PGMT in this work is similar to the Petrov-Galerkin method studied by Lin et al. [2] for linear Volterra integro-differential equations. The PGMT is a hybrid of the continuous and discontinuous Galerkin methods with respect to time. The trial space is the same as of that the continuous Galerkin method and the test space is a duplication of the test space in the discontinuous Galerkin method. Because of the discontinuity of the test space, the present method is highly stable. Theoretically, if the approximate solution is a piecewise linear polynomial in time, optimal order convergence results using $\mathrm{L}_{\infty}$-norm in time and the norm $\|\cdot\|_{\ell}$ in space (for $\ell=0,1$ ) have been shown globally of the PGFEM. Proving the convergence in the $\mathrm{L}_{\infty}$-norm in time and the norm $\|\cdot\|_{1}$ in space is a marked theoretical advantage over the other existing numerical methods for solving (1). Practically, the number of unknowns in the PGMT method is fewer than the number of unknowns in the discontinuous Galerkin method with respect to time. 
By setting $\tilde{\mathfrak{u}}(\mathrm{t})=\mathfrak{u}(\mathrm{t})-\mathfrak{u}(0)$, equation (1) becomes

$$
\tilde{u}_{t}+A \tilde{u}(t)+\int_{0}^{t} b(t, s) A \tilde{u}(s) d s=f(t)-A u(0)-\int_{0}^{t} b(t, s) A u(0) d s
$$

for $t \in(0, T]$ with $\tilde{u}(0)=0$. Therefore, without loss of generality, instead of (1), we consider the problem

$$
u_{t}+A u(t)+\int_{0}^{t} b(t, s) A u(s) d s=f(t) \quad \text { for } t \in(0, T] \text { with } u(0)=0 .
$$

To define our time PGMT, we introduce the time partitions: $0=t_{0}<t_{1}<$ $\cdots<t_{N}=T$ of $[0, T]$ and denote the step sizes by $k_{n}=t_{n}-t_{n-1}$. We set the time intervals $I_{n}=\left(t_{n-1}, t_{n}\right]$, and the maximum step size $k=\max _{n} k_{n}$. For $m \geq 1$, let $P_{m}\left(D\left(A^{1 / 2}\right)\right)$ denote the space of polynomials in the variable $t$ of degree strictly less than $m$ with coefficients in $D\left(A^{1 / 2}\right)$. Let

$$
\begin{aligned}
\mathcal{W}_{k}\left(D\left(A^{1 / 2}\right)\right):= & \left\{X \in C\left([0, T], D\left(A^{1 / 2}\right)\right): X(0)=0,\right. \\
& \left.\left.X\right|_{I_{n}} \in P_{2}\left(D\left(A^{1 / 2}\right)\right), 1 \leq n \leq N\right\}, \\
\mathcal{T}_{k}\left(D\left(A^{1 / 2}\right)\right):= & \left\{X \in L^{2}\left([0, T], D\left(A^{1 / 2}\right)\right):\left.X\right|_{I_{n}} \in P_{1}\left(D\left(A^{1 / 2}\right)\right), 1 \leq n \leq N\right\},
\end{aligned}
$$

be the trial and test spaces respectively.

The PGMT of problem (3) is: compute $U:[0, T] \times \Omega \rightarrow \mathcal{W}_{k}\left(D\left(A^{1 / 2}\right)\right)$ such that

$$
G_{N}(U, X)=\int_{0}^{T}\langle f(t), X(t)\rangle d t \quad \text { for all } X \in \mathcal{T}_{k}\left(D\left(A^{1 / 2}\right)\right)
$$

where

$$
\begin{aligned}
\mathrm{G}_{N}(\mathrm{U}, X)= & \int_{0}^{T}\left[\left\langle\mathrm{U}^{\prime}(\mathrm{t}), \mathrm{X}(\mathrm{t})\right\rangle+\mathrm{A}(\mathrm{U}(\mathrm{t}), \mathrm{X}(\mathrm{t}))\right] \mathrm{dt} \\
& +\int_{0}^{T} \int_{0}^{t} \mathrm{~b}(\mathrm{t}, \mathrm{s}) A(\mathrm{U}(\mathrm{s}), \mathrm{X}(\mathrm{t})) \mathrm{ds} d \mathrm{t},
\end{aligned}
$$


and $A(u, w)$ denote the bilinear forms on $D\left(A^{1 / 2}\right)$ generated by $\langle A u, w\rangle$.

The solution $u$ of $(3)$ satisfies $G_{N}(u, X)=\int_{0}^{t_{N}}\langle f(t), X(t)\rangle d t$ for all $X \in$ $\mathcal{T}_{k}\left(D\left(A^{1 / 2}\right)\right)$. So $u-u$ satisfies the orthogonality condition

$$
\mathrm{G}_{\mathrm{N}}(\mathrm{U}-\mathrm{u}, \mathrm{X})=0 \text { for all } \mathrm{X} \in \mathcal{T}_{\mathrm{k}}\left(\mathrm{D}\left(\mathrm{A}^{1 / 2}\right)\right) .
$$

Because the function $X$ in $\mathcal{T}_{k}\left(D\left(A^{1 / 2}\right)\right)$ is not required to be continuous at the nodes $t_{n}$ for $1 \leq n \leq N$, we choose its values on the different time intervals independently. By choosing $X$ to vanish outside $I_{n}$, our numerical scheme (4) reduces to one equation for each $I_{n}$ for $1 \leq n \leq N$. Therefore, the scheme (4) requires us to determine $\mathrm{U} \in \mathcal{W}_{\mathrm{k}}\left(\mathrm{D}\left(\mathrm{A}^{1 / 2}\right)\right)$ such that, for $\mathrm{n}=1, \ldots, \mathrm{N}$,

$$
\begin{aligned}
& \int_{t_{n-1}}^{t_{n}}\left[\left\langle u^{\prime}(t), X(t)\right\rangle+A(u(t), X(t))\right] d t+\int_{t_{n-1}}^{t_{n}} \int_{0}^{t} b(t, s) A(U(s), X(t)) d s d t \\
& =\int_{t_{n-1}}^{t_{n}}\langle f(t), X(t)\rangle d t \quad \text { for all } X \in P_{1}\left(D\left(A^{1 / 2}\right)\right)
\end{aligned}
$$

For the fully discrete scheme we assume that $\mathbb{H}=\mathrm{L}_{2}(\Omega)$ for a bounded, convex domain $\Omega$, and that $A$ is a strongly elliptic, second order partial differential operator subject to homogeneous Dirichlet boundary conditions. We also assume that $\mathbb{H}^{r}(\Omega)=\mathrm{H}^{r}(\Omega)$ and $\mathbb{H}_{0}^{1}(\Omega)=\mathrm{H}_{0}^{1}(\Omega)$ denotes the space of all functions $\phi \in \mathrm{H}^{1}(\Omega)$ with $\phi=0$ on $\partial \Omega$.

To describe our fully discrete scheme, we triangulate and define the usual continuous, piecewise linear finite element space $S_{h} \subseteq \mathbb{H}_{0}^{1}(\Omega)$, where $h$ denotes the maximum diameter of the elements. Let the mesh be quasi-uniform so that the Ritz projector $R_{h}: \mathbb{H}_{0}^{1}(\Omega) \rightarrow S_{h}$ for the positive definite bilinear form $A(u, v)$ which is defined by $A\left(R_{h} v-v, \chi\right)=0$ for all $\chi \in S_{h}$, has the approximation property

$$
\left\|v-\mathrm{R}_{\mathrm{h}} v\right\|_{\ell} \leq \mathrm{Ch}^{\mathrm{r}-\ell}\|v\|_{\mathrm{r}} \quad \text { for } v \in \mathbb{H}_{0}^{1}(\Omega) \cap \mathbb{H}^{\mathrm{r}}(\Omega) \quad \text { with } \mathrm{r} \geq 2,
$$


where $\|v\|_{m}:=\|v\|_{\mathbb{H}^{m}(\Omega)}=\|v\|_{H^{m}(\Omega)}$. Let $\mathrm{P}_{2}\left(S_{h}\right)$ and $\mathrm{P}_{1}\left(S_{h}\right)$ denote the spaces of polynomials in the variable $t$ of degree strictly less than 2 and 1 respectively with coefficients in $S_{h}$. Define the corresponding trial and test spaces of piecewise polynomials $\mathcal{W}_{k}\left(S_{h}\right)$ and $\mathcal{T}_{k}\left(S_{h}\right)$ respectively.

By combining the PGMT introduced above with the use of finite elements for the approximation in the spatial variables, we arrive at a fully discrete PGFEM of problem (3) which is: compute $\mathrm{U}_{\mathrm{h}}:[0, \mathrm{~T}] \times \Omega \rightarrow \mathcal{W}_{\mathrm{k}}\left(\mathrm{S}_{\mathrm{h}}\right)$ such that

$$
G_{N}\left(U_{h}, X\right)=\int_{0}^{t_{N}}\langle f(t), X(t)\rangle d t \quad \text { for all } X \in \mathcal{T}_{k}\left(S_{h}\right) .
$$

The next section, $\S 2$, proves the stability of the proposed numerical schemes. Optimal order convergence using $\mathrm{L}_{\infty}$-norm in time and $\mathbb{H}^{\ell}$-norm in space is shown in Section 3 for $\ell=0,1$. Some numerical experiments demonstrating our theoretical results are given in Section 4

\section{$2 \quad$ Stability results}

We study next the stability of the approximate solution $\mathrm{U}$ defined by (4) or equivalently by (7). Since (4) and (9) have the same form, the stability of the fully discrete solution $\boldsymbol{U}_{h}$ defined by (9) can be obtained in a similar manner.

Throughout the rest of the article, we assume that $b(t, t)=0$; however, our approach to prove the stability and the convergence can be extended to cover the case that $b(t, t)$ is not zero. In the next theorem we show the stability of the approximate solution $\mathrm{U}$. For brevity, we introduce the following notations

$$
\mathrm{U}^{\mathrm{n}}=\mathrm{U}\left(\mathrm{t}_{\mathrm{n}}\right), \quad\|\mathrm{U}\|_{J_{\mathfrak{n}}}=\sup _{\mathrm{t} \in \mathrm{J}_{\mathfrak{n}}}\|\mathrm{U}(\mathrm{t})\| \quad \text { and }
$$




$$
\|\mathrm{U}\|_{p, J_{n}}=\sup _{t \in J_{n}}\|\mathrm{U}(\mathrm{t})\|_{p} \quad \text { where } \quad J_{n}=\bigcup_{j=1}^{n} I_{j}
$$

Theorem 1 Given $\mathrm{U}^{0} \in \mathbb{H}$ and $\mathrm{f} \in \mathrm{L}_{2}((0, \mathrm{~T}) ; \mathbb{H})$, then the approximate solution $\mathrm{U}$ defined by (4) is stable and

$$
\|\mathrm{U}\|_{1, J_{n}}^{2} \leq \mathrm{C} \int_{0}^{t_{n}}\|\mathrm{f}(\mathrm{t})\|^{2} \mathrm{dt} \quad \text { for } \mathrm{n}=1,2, \ldots, \mathrm{N} .
$$

Proof: Choose $X=U^{\prime}$ in (4) and noting that $\left\langle\mathrm{U}^{\prime}(\mathrm{t}), \mathrm{U}^{\prime}(\mathrm{t})\right\rangle=\frac{1}{2}\left\|\mathrm{U}^{\prime}(\mathrm{t})\right\|^{2} \geq$ 0 and $A\left(U(t), U^{\prime}(t)\right)=\frac{1}{2} \frac{d}{d t} A(U(t), U(t))$, we have

$$
\begin{aligned}
& \int_{t_{j-1}}^{t_{j}}\left\|U^{\prime}(t)\right\|^{2} d t+A\left(U^{j}, U^{j}\right)-A\left(U^{j-1}, u^{j-1}\right) \\
& +2 \int_{t_{j-1}}^{t_{j}} \int_{0}^{t} b(t, s) A\left(U(s), U^{\prime}(t)\right) d s d t=2 \int_{t_{j-1}}^{t_{j}}\left\langle f(t), U^{\prime}(t)\right\rangle d t .
\end{aligned}
$$

Hence, summing from $\boldsymbol{j}=1$ to $j=n$ and using the Cauchy-Schwarz inequality, we find that

$$
\begin{aligned}
& \int_{0}^{t_{n}}\left\|U^{\prime}(t)\right\|^{2} d t+\left\|U^{n}\right\|_{1}^{2}=2 \int_{0}^{t_{n}}\left\langle f(t), U^{\prime}(t)\right\rangle d t-2 \mathcal{I}_{n} \\
\leq & \left.2 \int_{0}^{t_{n}}\|f(t)\|\left\|U^{\prime}(t)\right\| d t-2 \mathcal{I}_{n} \leq \int_{0}^{t_{n}}\|f(t)\|^{2} d t+\int_{0}^{t_{n}} \| U^{\prime}(t)\right) \|^{2} d t-2 \mathcal{I}_{n},
\end{aligned}
$$

where

$$
\mathcal{I}_{n}=\int_{0}^{t_{n}} \int_{0}^{t} b(t, s) A\left(u(s), u^{\prime}(t)\right) d s d t .
$$

Reversing the order of integrals, integrating by parts, and using $b(t, t)=0$ and $\mathrm{U}^{0}=0$, we obtain

$$
\mathcal{I}_{n}=\int_{0}^{t_{n}} \int_{s}^{t_{n}}\left\langle b(t, s) A u(s), u^{\prime}(t)\right\rangle d t d s
$$




$$
\begin{aligned}
= & \int_{0}^{t_{n}}\left(\left\langle b\left(t_{n}, s\right) A u(s), u\left(t_{n}\right)\right\rangle-\int_{s}^{t_{n}}\left\langle b_{t}(t, s) A u(s), u(t)\right\rangle d t\right) d s \\
= & \int_{0}^{t_{n}}\left\langle b\left(t_{n}, s\right) \frac{d}{d s} \int_{0}^{s} A u(q) d q, u\left(t_{n}\right)\right\rangle d s \\
& -\int_{0}^{t_{n}} \int_{0}^{t}\left\langle b_{t}(t, s) A u(s), u(t)\right\rangle d s d t \\
= & -\int_{0}^{t_{n}} \int_{0}^{s}\left\langle b_{s}\left(t_{n}, s\right) A u(q), u\left(t_{n}\right)\right\rangle d q d s \\
& -\int_{0}^{t_{n}} \int_{0}^{t}\left\langle b_{t}(t, s) A u(s), u(t)\right\rangle d s d t .
\end{aligned}
$$

Hence, using (2), we find that

$$
\begin{aligned}
\left|\mathcal{I}_{n}\right| \leq & C \int_{0}^{t_{n}}\left(t_{n}-t\right)^{\alpha-1} \int_{0}^{t}\|\mathrm{U}(\mathrm{s})\|_{1}\left\|\mathrm{U}\left(\mathrm{t}_{\mathrm{n}}\right)\right\|_{1} \mathrm{ds} d \mathrm{t} \\
& +\mathrm{C} \int_{0}^{t_{n}}\|\mathrm{U}(\mathrm{t})\|_{1} \int_{0}^{t}(\mathrm{t}-\mathrm{s})^{\alpha-1}\|\mathrm{U}(\mathrm{s})\|_{1} \mathrm{ds} d t
\end{aligned}
$$

and thus, the $\epsilon$ inequality and Lemma 6.3 of Larsson et al. [1] yield

$$
\begin{aligned}
\left|\mathcal{I}_{n}\right| \leq & \epsilon_{1} t_{n}^{\alpha+1}\left\|\mathrm{U}\left(\mathrm{t}_{n}\right)\right\|_{1}^{2}+\mathrm{C}_{\epsilon_{1}} \int_{0}^{t_{n}}\left(t_{n}-t\right)^{\alpha-1} \int_{0}^{t}\|\mathrm{U}(\mathrm{s})\|_{1}^{2} \mathrm{ds} d \mathrm{t} \\
& +\epsilon_{2} \int_{0}^{t_{n}}\|\mathrm{U}(\mathrm{t})\|_{1}^{2} \mathrm{dt}+\mathrm{C}_{\epsilon_{2}} \int_{0}^{t_{n}}\left(\int_{0}^{t}(t-s)^{\alpha-1}\|\mathrm{U}(\mathrm{s})\|_{1} d s\right)^{2} d t \\
\leq & \epsilon_{1} t_{n}^{\alpha+1}\left\|\mathrm{U}\left(\mathrm{t}_{\mathrm{n}}\right)\right\|_{1}^{2}+\epsilon_{2} \int_{0}^{t_{n}}\|\mathrm{U}(\mathrm{t})\|_{1}^{2} d t \\
& +\mathrm{C}_{\epsilon_{1}, \epsilon_{2}} \int_{0}^{t_{n}}\left(t_{n}-t\right)^{\alpha-1} \int_{0}^{t}\|\mathrm{U}(s)\|_{1}^{2} d s d t .
\end{aligned}
$$

Inserting this bound in (10) and choosing $\epsilon_{1}$ sufficiently small, we obtain

$$
\left\|U^{n}\right\|_{1}^{2} \leq C \int_{0}^{t_{n}}\|f(t)\|^{2} d t+2 \epsilon_{2} \int_{0}^{t_{n}}\|U(t)\|_{1}^{2} d t
$$




$$
+C_{\epsilon_{2}} \int_{0}^{t_{n}}\left(t_{n}-t\right)^{\alpha-1} \int_{0}^{t}\|U(s)\|_{1}^{2} d s d t .
$$

Since $\|\mathrm{U}\|_{1, \mathrm{~J}_{\mathrm{n}}}=\left\|\mathrm{U}^{\mathrm{n}_{\mathrm{o}}}\right\|_{1}$ for some $1 \leq \mathrm{n}_{0} \leq \mathrm{n}$, and so by using (11) for $\mathrm{n}=\mathrm{n}_{0}$, we achieve

$$
\begin{aligned}
\int_{0}^{t_{n_{0}}}\|\mathrm{U}(\mathrm{t})\|_{1}^{2} d t \leq t_{n_{0}}\left\|U^{n_{0}}\right\|_{1}^{2} \leq & C t_{n_{0}} \int_{0}^{t_{n_{0}}}\|f(t)\|^{2} d t+2 t_{n} \epsilon_{2} \int_{0}^{t_{n_{0}}}\|\mathrm{U}(t)\|_{1}^{2} d t \\
& +C_{\epsilon_{2}} t_{n_{0}} \int_{0}^{t_{n_{0}}}\left(t_{n_{0}}-t\right)^{\alpha-1} \int_{0}^{t}\|\mathrm{U}(s)\|_{1}^{2} d s d t .
\end{aligned}
$$

Thus, for $\epsilon_{2}$ sufficiently small, we have

$\int_{0}^{t_{n_{0}}}\|\mathrm{U}(\mathrm{t})\|_{1}^{2} \mathrm{dt} \leq \mathrm{C} \int_{0}^{t_{n_{0}}}\|f(t)\|^{2} d t+C \int_{0}^{t_{n_{0}}}\left(t_{n_{0}}-t\right)^{\alpha-1} d t \int_{0}^{t_{n_{0}}}\|\mathrm{U}(s)\|_{1}^{2} d s$.

Now, an application of Lemma 6.4 of Larsson et al. [1] yields $\int_{0}^{t_{n_{0}}}\|\mathrm{U}(\mathrm{t})\|_{1}^{2} \mathrm{dt} \leq$ $C \int_{0}^{t_{n_{0}}}\|f(t)\|^{2} d t$. Inserting this into (11), we find that

$$
\begin{aligned}
\|\mathrm{U}\|_{1, J_{n}}^{2} & =\left\|\mathrm{U}^{n_{0}}\right\|_{1, J_{n}}^{2} \\
& \leq C \int_{0}^{t_{n_{0}}}\|f(t)\|^{2} d t+C \int_{0}^{t_{n}}\left(t_{n_{0}}-t\right)^{\alpha-1} \int_{0}^{t_{n_{0}}}\|f(s)\|^{2} d s d t \\
& \leq C \int_{0}^{t_{n_{0}}}\|f(s)\|^{2} d s \leq C \int_{0}^{t_{n}}\|f(s)\|^{2} d s .
\end{aligned}
$$

\section{$3 \quad$ Error estimates}

We estimate the errors $\mathrm{U}-\mathfrak{u}$ and $\mathrm{U}_{\mathrm{h}}-\mathfrak{u}$ where $\mathrm{U}$ and $\mathrm{U}_{\mathrm{h}}$ are the approximate solutions obtained using the PGMT and PGFEM respectively. In the next two 
theorems we assume that there exists a constant $C_{0}$ such that

$$
\left\|u^{\prime \prime}\right\|_{\ell, J_{n}}+\int_{0}^{t_{n}}\left\|A u^{\prime \prime}(t)\right\|^{2} d t \leq C_{0} \quad \text { for all } 1 \leq n \leq N .
$$

In the following theorem we derive the error $\mathbf{U}-\mathfrak{u}$ when $\boldsymbol{U}$ is given by (4) and $\boldsymbol{u}$ is the continuous solution of (3).

Theorem 2 Let $\mathrm{U}$ and $\mathrm{u}$ be the solutions of (4) and (3) respectively. Then

$$
\|\mathrm{U}-\mathrm{u}\|_{\ell, \mathrm{J}_{\mathrm{n}}} \leq \mathrm{Ck}^{2} \quad \text { for } \ell=0,1 \text { and for all } 1 \leq \mathrm{n} \leq \mathrm{N} .
$$

Proof: Let $\Pi u \in \mathcal{W}_{k}\left(D\left(A^{1 / 2}\right)\right)$ be a continuous piecewise linear interpolant of $u$ defined by

$$
\Pi u\left(t_{n}\right)=u\left(t_{n}\right) \text { for } n=0,1, \ldots, N .
$$

Put $\theta=u-\Pi u$ and $\eta=\Pi u-u$. We decompose the error into two terms,

$$
u-u=\theta+\eta,
$$

and estimate each term separately. For $\ell=0,1$,

$$
\|\eta\|_{\ell, J_{n}} \leq \mathrm{C} \max _{1 \leq j \leq n}\left(k_{n} \int_{t_{j-1}}^{t_{j}}\left\|u^{\prime \prime}(t)\right\|_{\ell} d t\right) \leq C k^{2}\left\|u^{\prime \prime}\right\|_{\ell, J_{n}} \leq C k^{2} .
$$

To estimate the term $\theta$ in (12), we use the orthogonality relation (6) and get

$$
\mathrm{G}_{\mathrm{N}}(\theta, X)=-\mathrm{G}_{\mathrm{N}}(\eta, X) \quad \text { for } X \in \mathcal{T}_{k}\left(D\left(A^{1 / 2}\right)\right) \quad \text { with } 1 \leq n \leq N .
$$

Using the defining properties of $\Pi u$ and $X$ is constant on $I_{n}$, we conclude

$$
\mathrm{G}_{N}(\theta, X)=-\int_{0}^{t_{N}}\left\langle A \eta(t)+\int_{0}^{t} b(t, s) A \eta(s) d s, X(t)\right\rangle d t
$$


for all $X \in \mathcal{T}_{k}\left(D\left(A^{1 / 2}\right)\right)$. Equation (14) has the same form as the equation (4) which is satisfied by $\mathrm{U}$, so we apply the stability result of Theorem 1 and obtain

$$
\|\theta\|_{1, J_{n}}^{2} \leq C \int_{0}^{t_{n}}\left(\|A \eta(t)\|+\int_{0}^{t}|b(t, s)|\|A \eta(s)\| d s\right)^{2} \quad \text { for } 1 \leq n \leq N .
$$

The Cauchy-Schwarz inequality, (2), Lemma 6.3 of Larsson et el. [1], and (13), yield

$$
\begin{aligned}
\|\theta\|_{1, J_{n}}^{2} & \leq 2 \int_{0}^{t_{n}}\|A \eta(t)\|^{2} d t+C \int_{0}^{t_{n}}\left(\int_{0}^{t}(t-s)^{\alpha-1}\|A \eta(s)\| d s\right)^{2} d t \\
& \leq 2 \int_{0}^{t_{n}}\|A \eta(t)\|^{2} d t+C \int_{0}^{t_{n}}\left(t_{n}-t\right)^{\alpha-1} \int_{0}^{t}\|A \eta(s)\|^{2} d s d t \\
& \leq C \sum_{j=1}^{n} \int_{t_{j-1}}^{t_{j}}\|A \eta(t)\|^{2} d t \\
& \leq C \sum_{j=1}^{n} k_{j}\left(k_{j} \int_{t_{j-1}}^{t_{j}}\left\|A u^{\prime \prime}(s)\right\| d s\right)^{2} d t \\
& \leq C \sum_{j=1}^{n} k_{j}^{4} \int_{t_{j-1}}^{t_{j}}\left\|A u^{\prime \prime}(s)\right\|^{2} d s \\
& \leq C k^{4} .
\end{aligned}
$$

Therefore, using (12), (13), and (15), we obtain the desired result.

Next we estimate the error $\mathbf{U}_{h}-\mathfrak{u}$ where $\boldsymbol{U}_{h}$ is the approximate solution obtained using the PGFEM and $u$ is the continuous solution of (3).

Theorem 3 If $\mathrm{u}$ is the solution of the problem (3) and if $\mathrm{U}_{\mathrm{h}}$ is the approximate solution defined by (9), then for $\mathrm{r} \geq 2$ and $\ell=0,1$,

$$
\left\|\mathrm{U}_{\mathrm{h}}-\mathrm{u}\right\|_{\ell, \mathrm{J}_{\mathrm{n}}} \leq \mathrm{C} k^{2}+\mathrm{Ch}^{\mathrm{r}-\ell}\left(\int_{0}^{t_{n}}\left\|\mathrm{u}^{\prime}(\mathrm{t})\right\|_{\mathrm{r}}^{2} \mathrm{dt}\right)^{1 / 2} \quad \text { for all } 1 \leq \mathrm{n} \leq \mathrm{N} .
$$


Proof: We use the Ritz projector $R_{h}$ and decompose the error as

$$
\mathrm{U}_{\mathrm{h}}-\mathrm{u}=\left(\mathrm{U}_{\mathrm{h}}-\Pi \mathrm{R}_{\mathrm{h}} \mathrm{u}\right)+\left(\Pi \mathrm{R}_{\mathrm{h}} \mathrm{u}-\mathrm{u}\right) .
$$

So,

$$
\left\|\mathrm{U}_{\mathrm{h}}-\mathrm{u}\right\|_{\ell, \mathrm{J}_{\mathrm{n}}} \leq\left\|\Pi \mathrm{R}_{\mathrm{h}} \mathrm{u}-\mathrm{u}\right\|_{\ell, \mathrm{J}_{\mathfrak{n}}}+\left\|\mathrm{u}_{\mathrm{h}}-\Pi \mathrm{R}_{\mathrm{h}} \mathrm{u}\right\|_{\ell, \mathrm{J}_{\mathfrak{n}}} \quad \text { for } \ell=0,1 .
$$

To estimate the first term $\Pi \boldsymbol{R}_{h} \mathfrak{u}-\mathfrak{u}$, we write $\xi=\boldsymbol{R}_{h} \mathfrak{u}-\mathfrak{u}$, using (13), $\xi(0)=0$, and (8) with $\mathfrak{u}^{\prime}$ in place of $v$, we observe that for $\ell=0,1$,

$$
\begin{aligned}
\left\|\Pi R_{h} u-u\right\|_{\ell, J_{n}} & =\left\|\Pi u-u+\Pi\left(R_{h} u-u\right)\right\|_{\ell, J_{n}} \\
& \leq\|\Pi u-u\|_{\ell, J_{n}}+\|\xi\|_{\ell, J_{n}} \\
& \leq\|\Pi u-u\|_{\ell, J_{n}}+\|\xi(0)\|_{\ell}+\int_{0}^{t_{n}}\left\|\xi^{\prime}\right\|_{\ell} d t \\
& \leq C k^{2}+\mathrm{Ch}^{r-\ell} \int_{0}^{t_{n}}\left\|u^{\prime}(t)\right\|_{r} d t .
\end{aligned}
$$

The Galerkin orthogonality property (6) now takes the form

$$
\mathrm{G}_{\mathrm{N}}\left(\mathrm{U}_{\mathrm{h}}-\mathrm{u}, \mathrm{X}\right)=0 \quad \text { for all } \mathrm{X} \in \mathcal{T}_{\mathrm{k}}\left(\mathrm{S}_{\mathrm{h}}\right)
$$

Adapting the proof of Theorem 2, we see from (19) that

$$
G_{N}\left(U_{h}-V, X\right)=-G_{N}(V-u, X) \quad \text { for all } X \in \mathcal{T}_{k}\left(S_{h}\right),
$$

where $\mathrm{V}:=\Pi \mathrm{R}_{\mathrm{h}} \mathrm{u}$, and because

$$
\begin{aligned}
\int_{t_{n-1}}^{t_{n}}\left\langle V^{\prime}, X\right\rangle d t & =\left\langle\Pi R_{h} u\left(t_{n}\right)-\Pi R_{h} u\left(t_{n-1}\right), X\left(t_{n-1 / 2}\right)\right\rangle \\
& =\left\langle R_{h} u\left(t_{n}\right)-R_{h} u\left(t_{n-1}\right), X\left(t_{n-1 / 2}\right)\right\rangle \\
& =\int_{t_{n-1}}^{t_{n}}\left\langle\left(R_{h} u\right)^{\prime}, X\right\rangle d t=\int_{t_{n-1}}^{t_{n}}\left\langle R_{h} u^{\prime}, X\right\rangle d t
\end{aligned}
$$


the formula (5) gives

$\mathrm{G}_{\mathrm{N}}(\mathrm{V}-\mathrm{u}, \mathrm{X})=\sum_{n=1}^{N} \int_{t_{n-1}}^{t_{n}}\left\langle\xi^{\prime}+A(V-u)+\int_{0}^{t} b(t, s) A(V-u)(s) d s, X\right\rangle d t$.

The definition of the Ritz projector gives

$$
A(V-u, X)=A\left(R_{h} \Pi u-u, X\right)=A(\Pi u-u, X)=\langle A \eta, X\rangle,
$$

SO

$$
\mathrm{G}_{\mathrm{N}}(V-u, X)=\int_{0}^{t_{N}}\left\langle\xi^{\prime}+A \eta+\int_{0}^{t} b(t, s) A \eta(s) d s, X\right\rangle d t .
$$

Thus, from (20),

$$
\mathrm{G}_{N}\left(\mathrm{U}_{\mathrm{h}}-\mathrm{V}, X\right)=-\int_{0}^{t_{N}}\left\langle\xi^{\prime}+A \eta+\int_{0}^{t} \mathrm{~b}(\mathrm{t}, \mathrm{s}) A \eta(s) d s, X\right\rangle d t .
$$

for all $X \in \mathcal{W}_{k}\left(S_{h}\right)$. Stability of the PGMT (Theorem 1 with $\mathbb{H}=S_{h}$ ) and the already estimated term $\int_{0}^{t_{n}}\left\|A \eta+\int_{0}^{t} b(t, s) A \eta(s) d s\right\|^{2} d t$ in Theorem 2 now yield the estimate

$$
\begin{aligned}
\left\|\mathrm{U}_{\mathrm{h}}-\mathrm{V}\right\|_{1, J_{n}}^{2} & \leq \mathrm{C} \int_{0}^{t_{n}}\left(\left\|\xi^{\prime}\right\|^{2}+\left\|A \eta+\int_{0}^{t} \mathrm{~b}(\mathrm{t}, \mathrm{s}) A \eta(s) \mathrm{ds}\right\|^{2}\right) d t \\
& \leq C k^{4}+C h^{2 r} \int_{0}^{t_{n}}\left\|u^{\prime}(t)\right\|_{r}^{2} d t .
\end{aligned}
$$

Inserting (21) in (17) and using (18) we complete the proof.

\section{Numerical experiments}

We apply the fully discrete PGFEM (9) to solve a one dimensional problem of the form (3) over the time interval $[0,1]$. Our approximate solution is 


\begin{tabular}{|r|ll|ll|ll|}
\hline & \multicolumn{2}{|c|}{$\mathrm{N}=\mathrm{M}$} & \multicolumn{2}{c|}{$\mathrm{N}=\mathrm{M}$} & \multicolumn{2}{c|}{$\mathrm{N}=\left[\mathrm{M}^{1 / 2}\right]$} \\
$\mathrm{M}$ & $\left\|\mathrm{U}_{\mathrm{h}}-\mathrm{u}\right\|_{\mathrm{L}_{\infty}\left(\mathrm{L}_{2}\right)}$ & $\left\|\mathrm{U}_{\mathrm{h}}-\mathrm{u}\right\|_{\mathrm{L}_{\infty}\left(\mathrm{H}^{1}\right)}$ & $\left\|\mathrm{U}_{\mathrm{h}}-\mathrm{u}\right\|_{\mathrm{L}_{\infty}\left(\mathrm{H}^{1}\right)}$ \\
\hline 40 & $1.6 \mathrm{e}-03$ & & $2.0 \mathrm{e}-01$ & & $2.0 \mathrm{e}-01$ & \\
80 & $4.0 \mathrm{e}-04$ & 1.999 & $1.0 \mathrm{e}-01$ & 0.999 & $1.0 \mathrm{e}-01$ & 0.999 \\
160 & $1.0 \mathrm{e}-04$ & 2.006 & $5.0 \mathrm{e}-02$ & 0.999 & $5.0 \mathrm{e}-02$ & 1.002 \\
320 & $2.2 \mathrm{e}-05$ & 2.121 & $2.5 \mathrm{e}-02$ & 0.999 & $2.5 \mathrm{e}-02$ & 0.999 \\
640 & $5.7 \mathrm{e}-06$ & 2.120 & $1.2 \mathrm{e}-02$ & 0.999 & $1.2 \mathrm{e}-02$ & 1.001 \\
\hline
\end{tabular}

TABLE 1: The errors $\left\|U_{h}-u\right\|_{L_{\infty}\left(\mathrm{L}_{2}\right)}\left(=\left\|\mathrm{U}_{h}-\mathfrak{u}\right\|_{0, J_{N}}\right)$ and $\left\|U_{h}-u\right\|_{L_{\infty}\left(H^{1}\right)}$ $\left(=\left\|\mathrm{U}_{\mathrm{h}}-\mathfrak{u}\right\|_{1, \mathrm{~J}_{\mathrm{N}}}\right)$ and the order of convergence using a uniform mesh in both time and space consisting of $N$ and $M$ subintervals respectively.

a piecewise linear polynomial in the time and space variables, so $r=2$ in Theorem 3.

We let $\Omega=(0,1), A u=-2 u_{x x}$, and for $0 \leq s \leq t \leq 1, b(t, s)=$ $(t-s)^{\alpha} / \Gamma(\alpha+1)$, where $\Gamma$ is the standard gamma function and $\alpha=0.7$. We assume that $\mathfrak{u}=\mathfrak{u}(x, t)$ satisfies homogeneous Dirichlet boundary conditions $u(0, t)=0=u(1, t)$ for all $t \in[0,1]$. We choose $f(x, t)$ such that $u=$ $-t^{\alpha+1} \sin (2 \pi x)$ is the exact solution of (3). Thus $f(x, t)=-\left[(\alpha+1) t^{\alpha}+8 \pi^{2} t^{\alpha+1}+4 \pi^{2} t^{2(\alpha+1)} \Gamma(\alpha+2) / \Gamma(2 \alpha+3)\right] \sin (2 \pi x)$.

We see that the global error $\left\|\mathrm{U}_{\mathrm{h}}-\mathfrak{u}\right\|_{\ell, \mathrm{J}_{\mathrm{N}}}$ shown in Table 1 is of order $\mathrm{h}^{2-\ell}+\mathrm{k}^{2}$ for $\ell=0,1$, and so is consistent with our theoretical results in Theorem 3 .

Acknowledgements The support of the KFUPM is gratefully acknowledged. 


\section{References}

[1] S. Larsson, V. Thomée and L. Wahlbin, Numerical solution of parabolic integro-differential equations by the discontinuous Galerkin method, Math. Comp., 67, 45-71 (1998). C612, C617, C618, C620

[2] T. Lin, Y. Lin, M. Rao and S. Zhang, Petrov-Galerkin methods for linear Volterra integro-differential equations, SIAM J. Numer. Anal., 38, 937-963 (2000). doi:10.1137/S0036142999336145 C612

[3] W. McLean, I. H. Sloan and V. Thomée, Time discretization via Laplace transformation of an integro-differential equation of parabolic type, Numer. Math., 102, 497-522 (2006). C612

[4] N. Y. Zhang, On fully discrete Galerkin approximations for partial inregro-differential equations of parabolic type, Math. Comp., 60, 133-166 (1993). http://www. jstor.org/pss/2153159 C612

\section{Author address}

1. K. Mustapha, Department of Mathematics and Statistics, King Fahd University of Petroleum and Minerals, Dhahran, 31261, SAUdi Arabia.

mailto:kassem@kfupm.edu.sa 